\title{
ON THE SHAPE OF THE UNIT SPHERE IN $Q(\Delta)$
}

\author{
LISA R. GOLDBERG
}

(Communicated by Charles Pugh)

\begin{abstract}
We show that the unit sphere in the Banach space of $L^{1}$ holomorphic quadratic differentials on the disk is weakly uniformly convex with exponent $1 / 2$ at certain points.
\end{abstract}

\section{INTRODUCTION}

Recall that a holomorphic quadratic differential $\phi$ on a Riemann surface $R$ is an assignment of a holomorphic function $\phi_{i}\left(z_{i}\right)$ to each local coordinate $z_{i}$ on $R$, subject to the transformation law

$$
\phi_{i}\left(z_{i}\right) \cdot \frac{d z_{i}^{2}}{d z_{j}^{2}}=\phi_{j}\left(z_{j}\right)
$$

on overlapping coordinate neighborhoods. It follows that the area element $|\phi(z)||d z|^{2}$ is globally defined on $R$, and the $L^{1}$ norm of $\phi$ is

$$
|\phi|_{1}=\int_{R}|\phi(z)||d z|^{2} .
$$

With this norm, the $L^{1}$ holomorphic quadratic differentials on any Riemann surface $R$ form a Banach space which we denote $Q(R)$, and the unit sphere in $Q(R)$ is denoted $S(R)$. When $R$ is a Riemann surface of finite type, the space $Q(R)$ is the cotangent space to the Teichmüller space of marked conformal structures on $R$ [Ga].

We will focus on the special case when $R$ is equal to the open unit disk $\Delta$. Since $\Delta$ is contractible, $Q(\Delta)$ can be identified with the Banach space $L_{a}^{1}(\Delta)$ of holomorphic $L^{1}$-functions on $\Delta$. It is nevertheless advantageous to think of the elements of $Q(\Delta)$ as quadratic differentials. Specifically, there is an action on $Q(\Delta)$ by the Möbius group $\mathscr{M}$ of hyperbolic isometries of the disk defined by

$$
M^{*}(\phi)=\phi \circ M \cdot\left(M^{\prime}\right)^{2}
$$

which preserves the $L^{1}$ norm.

Received by the editors December 27, 1991.

1991 Mathematics Subject Classification. Primary 30C40, 30F60, 46A25, 46B10, 46B20.

This research is supported in part by the National Science Foundation program, Visiting Professorships for Women. 
Definition. A Banach space $X$ is smooth if its unit sphere $S$ is a smooth submanifold in the following weak sense: at every $x \in S$, the limit

$$
\lim _{t \rightarrow 0} \frac{|x+t y|-|x|}{t}
$$

exists for all directions $y \in S$. This limit is the Gateaux derivative of the norm at $x$ in the direction $y$, and it is written $x_{*}(y)$. In fact, $x_{*}$ extends to the unique linear functional on $X$ for which $x_{*}(x)=1$ [D].

Consider the (nonsymmetric) pairing $\langle\cdot, \cdot\rangle: S(\Delta) \times S(\Delta) \rightarrow \mathbf{R}$ defined by

$$
\langle\phi, \psi\rangle=\operatorname{Re} \int_{\Delta} \phi(z) \frac{\overline{\psi(z)}}{|\psi(z)|}|d z|^{2} .
$$

A straightforward calculation shows that

$$
\langle\phi, \psi\rangle=\phi_{*}(\psi)
$$

is the Gateaux derivative of $\phi$ in the direction $\psi$. Note that for $M \in \mathscr{M}$,

$$
\left\langle M^{*} \phi, M^{*} \psi\right\rangle=\langle\phi, \psi\rangle \text {. }
$$

Translating into local coordinates, the quantity $\langle\phi, \psi\rangle$ is equal to the cosine of the difference $\arg (\phi)-\arg (\psi)$ averaged over $R$ with respect to the area measure $|\phi(z)||d z|^{2}$. Consequently, if $\phi$ is $L^{1}$ close to $\psi$ then $\langle\phi, \psi\rangle$ is nearly 1 . Indeed,

$$
\begin{aligned}
\varepsilon & =|\psi-\phi|_{1} \geq \int_{\Delta}|\psi(z)-\phi(z)| \cos (\arg (\psi(z)-\phi(z))-\arg (\psi(z)))|d z|^{2} \\
& =\langle\psi-\phi, \psi\rangle=1-\langle\phi, \psi\rangle .
\end{aligned}
$$

The converse, however, does not hold.

Definition. A smooth Banach space $X$ is weakly uniformly convex at a point $x$ in the unit sphere $S$ if, for all directions $y \in S, x_{*}(y)=1-\varepsilon$ implies that $|x-y|_{1}=\delta$ where $\delta \rightarrow 0$ as $\varepsilon \rightarrow 0$. If $\delta$ can be chosen to be $O\left(\varepsilon^{\alpha}\right)$, then $X$ is weakly uniformly convex at $x \in S$ with exponent $\alpha$.

It is known that there are points in $S(\Delta)$ at which $Q(\Delta)$ is not uniformly convex. See [Mc, $\S 5]$ or $\S 3$ of this article. By contrast McMullen proves that $Q(\Delta)$ is weakly uniformly convex at the constant differential $\psi(z)=(1 / \pi) \cdot d z^{2}$ with exponent $\alpha<1 / 6$. Here, we improve his estimate:

Theorem. The Banach space $Q(\Delta)$ is weakly uniformly convex at $\psi=1 / \pi \cdot d z^{2}$ with exponent $1 / 2$.

Definition. A Banach space $X$ is uniformly convex at a point $x$ in the unit sphere if, for all $\varepsilon>0$, there exists $\delta>0$ such that

$$
|y+x|=2-\varepsilon \Rightarrow|y-x|<\delta .
$$

If $\delta$ can be chosen to be $O\left(\varepsilon^{\alpha}\right)$, then $X$ is uniformly convex at $x$ with exponent $\alpha$.

A Hilbert space is uniformly convex with exponent $1 / 2$ at every point. By contrast, $Q(\Delta)$ is nowhere uniformly convex, as is illustrated by the following example due to McMullen. 
Example. Fix $\psi \in S(\Delta), \varepsilon>0$, and choose $\phi \in S(\Delta)$ with the following property: there exist disjoint subsets $A, B \subset \Delta$ such that

$$
\int_{A}|\phi||d z|^{2}>1-\varepsilon \text { and } \int_{B}|\psi||d z|^{2}>1-\varepsilon
$$

This can be achieved by taking a sequence $M_{n}$ of Möbius transformations which tend to $\infty$ in $\mathscr{M}$ and setting $\phi=M_{n}^{*} \psi$ for $n$ sufficiently large. Then

$$
\begin{aligned}
|\phi \pm \psi|_{1} & \geq \int_{A}|\phi(z) \pm \psi(z)||d z|^{2}+\int_{B}|\phi(z) \pm \psi(z)||d z|^{2} \\
& >\int_{A}|\phi(z)||d z|^{2}-\int_{A}|\psi(z)||d z|^{2}+\int_{B}|\psi(z)||d z|^{2}-\int_{B}|\phi(z)||d z|^{2} \\
& =2-4 \varepsilon .
\end{aligned}
$$

This article is organized as follows: $\S 1$ recalls the construction of a particular bounded linear projection from $L^{1}(\Delta)$ to $L_{a}^{1}(\Delta)$. This projection is used in $\S 2$ to prove the theorem. The concluding $\S 3$ describes connections between $Q(\Delta)$ and the Bloch space and mentions several open problems.

\section{A REPRODUCING KERNEL}

The material in this section is standard; references are [A, Ga, FR].

Recall that the density function of the Poincare metric on the disk $\Delta$ is given by the formula

$$
\rho(z)=1 /\left(1-|z|^{2}\right)
$$

Let $K: \Delta \times \Delta \rightarrow \mathrm{C}$ be defined by

$$
K(z, w)=1 /(1-z \bar{w})^{4} .
$$

For each $z \in \Delta$,

$$
\sup _{w \in \Delta} \rho(w)^{-2}|K(z, w)|<\infty
$$

Therefore,

$$
T f(z)=\frac{3}{\pi} \cdot \int_{\Delta} \rho(w)^{-2} K(z, w) f(w)|d w|^{2}
$$

is a holomorphic function on the disk whenever $f \in L^{1}(\Delta)$.

For any $w$ and any Möbius transformation $M$,

$$
\int_{\Delta}|K(z, w)||d z|^{2}=\left|M^{\prime}(w)\right|^{2} \cdot \int_{\Delta}|K(z, M(w))||d z|^{2} .
$$

Since $\int_{\Delta}|K(z, 0)||d z|^{2}=\pi$, it follows that $\int_{\Delta}|K(z, w)||d z|^{2}=\rho(w)^{2}$. This fact, together with Fubini's theorem, implies that $T f \in L^{1}(\Delta)$ whenever $f$ is

$$
\begin{aligned}
\int_{\Delta}|T f(z)||d z|^{2} & =\frac{3}{\pi} \cdot \int_{\Delta}\left(\int_{\Delta}\left|\rho(w)^{-2} K(z, w) f(w)\right||d z|^{2}\right)|d w|^{2} \\
& \leq 3 \cdot \int_{\Delta}|f(w)||d w|^{2}
\end{aligned}
$$


We will need the following

Proposition [FR]. The transformation $T: L^{1}(\Delta) \rightarrow L_{a}^{1}(\Delta)$ is a bounded linear projection with norm at most 3 that maps antiholomorphic functions to constants. More precisely, when $\phi \in L_{a}^{1}(\Delta)$,

(1) $T \phi=\phi$,

(2) $T \bar{\phi}=\overline{\phi(0)}$.

Proof. It remains to prove statements (1) and (2). Since holomorphic functions satisfy the mean value property, we have

$$
\phi(0)=\frac{1}{2 \pi} \cdot \int_{|z|=r} \phi\left(r e^{2 \pi i \theta}\right) d \theta=\frac{3}{\pi} \cdot \int_{\Delta} \rho(w)^{-2} K(0, w) \phi(w)|d w|^{2}=T \phi(0) \text {. }
$$

Let $A \in \mathscr{M}$ be a Möbius transformation that takes 0 to $z$ and set

$$
\Phi(z)=\frac{A^{\prime}(z)^{2} \phi(A(z))}{A^{\prime}(0)^{2}} .
$$

Then,

$$
\phi(z)=\Phi(0)=\frac{3}{\pi} \cdot \int_{\Delta} \rho(w)^{-2} K(z, w) \phi(w)|d w|^{2}=T \phi(z),
$$

proving (1).

A second application of the mean value property (to antiholomorphic functions) yields

$$
\begin{aligned}
{\overline{\phi(0) A^{\prime}(0)}}^{2} & =\frac{3}{\pi} \cdot \int_{\Delta} \rho(w)^{-2}{\overline{\phi(w) A^{\prime}(w)}}^{2}|d w|^{2} \\
& ={\overline{A^{\prime}(0)}}^{2} \cdot \int_{\Delta} \rho(w)^{-2} K(z, w) \overline{\phi(w)}|d w|^{2}
\end{aligned}
$$

since ${\overline{A^{\prime}(w)}}^{2}={\overline{A^{\prime}(0)}}^{2} \cdot K(z, w)$. This shows (2).

Corollary. If $\phi \in L_{a}^{1}(\Delta)$ is normalized so that $\phi(0)=0$, then

Proof.

$$
\int_{\Delta}|\operatorname{Re} \phi(z)||d z|^{2} \leq 6 \cdot \int_{\Delta}|\operatorname{Im} \phi(z)||d z|^{2} .
$$

Therefore, $|\operatorname{Re} \phi|_{1} \leq|\phi|_{1}=2 \cdot|T(\operatorname{Im} \phi)|_{1} \leq 6 \cdot|\operatorname{Im} \phi(z)|_{1}$.

$$
T(\operatorname{Im} \phi)=\frac{-i}{2} \cdot T(\phi-\bar{\phi})=\frac{-i}{2} \cdot \phi
$$

2. THE EXPONENT OF WEAK UNIFORM CONVEXITY IS $1 / 2$

Proof of Theorem. We are given $\psi=1 / \pi \cdot d z^{2}$ and $\phi$ in $S(\Delta)$ satisfying

$$
\langle\psi, \phi\rangle=\int_{\Delta} \operatorname{Re} \phi(z)|d z|^{2}=1-\varepsilon
$$

This mean value theorem yields $\operatorname{Re} \phi(0)=(1-\varepsilon) / \pi$. Since $\int_{\Delta}|\phi(z)||d z|^{2}=1$, the Cauchy-Schwarz inequality implies

$$
\begin{aligned}
& \int_{\Delta}|\operatorname{Im} \phi(z)||d z|^{2}=\int_{\Delta}\left(|\phi(z)|^{2}-|\operatorname{Re} \phi(z)|^{2}\right)^{1 / 2}|d z|^{2} \\
& \quad \leq\left(\int_{\Delta}|\phi(z)|-|\operatorname{Re} \phi(z)||d z|^{2}\right)^{1 / 2} \cdot\left(\int_{\Delta}|\phi(z)|+|\operatorname{Re} \phi(z)||d z|^{2}\right)^{1 / 2} \\
& \leq \varepsilon^{1 / 2} \cdot 2^{1 / 2}
\end{aligned}
$$


Finally,

$$
\begin{aligned}
\mid \phi- & \left.\psi\right|_{1}=\int_{\Delta}\left|\frac{1}{\pi}-\phi(z)\right||d z|^{2} \\
& \leq \int_{\Delta}\left|\frac{1}{\pi}-\operatorname{Re} \phi(0)\right||d z|^{2}+\int_{\Delta}|\operatorname{Re} \phi(0)-\operatorname{Re} \phi(z)||d z|^{2}+\int_{\Delta}|\operatorname{Im} \phi(z)||d z|^{2} \\
& \leq \frac{\varepsilon}{\pi}+6 \cdot \int_{\Delta}|\operatorname{Im} \phi(0)-\operatorname{Im} \phi(z)||d z|^{2}+\varepsilon^{1 / 2} \cdot 2^{1 / 2} \\
& =O\left(\varepsilon^{1 / 2}\right)
\end{aligned}
$$

where the second inequality depends on the Corollary in $\S 1$. This completes the proof of the theorem.

Remark. The proof given above can be used to show that $Q(\Delta)$ is weakly uniformly convex with exponent $1 / 2$ at:

(1) all constant differentials $\left(e^{i \theta} / \pi\right) \cdot d z^{2}$, and

(2) all differentials $M^{*} \psi$ where $M \in \mathscr{M}$ and $\psi$ is a constant differential.

\section{OPEN QUESTIONS}

Below we list a few of the open questions that arise in this discussion.

Problem 1. Is $Q(\Delta)$ weakly uniformly convex at differentials $\phi=((n+2) / 2 \pi)$. $z^{n} d z^{2}$ ? at differentials $\phi \neq 0$ which have holomorphic nonzero extensions to a neighborhood of the closed disk?

Problem 2. Characterize the flat points in $S(\Delta)$ where $Q(\Delta)$ is not weakly uniformly convex.

For completeness, we include McMullen's construction of a flat point in $Q(\Delta)$ [Mc, §5].

Example. Select a sequence of points $z_{n} \in \Delta$ which are the centers of a family of disjoint hyperbolic disks whose radii tend to $\infty$. Let $M_{n}$ be a sequence of Möbius transformations such that $M_{n}\left(z_{n}\right)=0$. Let $\psi=1 / \pi \cdot d z^{2}, \psi_{n}=M_{n}^{*} \psi$, and

$$
\phi=c \cdot \sum_{n} 2^{-n} \cdot M_{n}^{*} \psi
$$

where the constant is chosen so that $\phi \in S(\Delta)$. Then $\left\langle\psi_{n}, \phi\right\rangle \rightarrow 1$; however, $\left|\psi_{n}-\phi\right|_{1}$ does $n o t$ tend to 0 .

Problem 3. Let $R$ be a finite type Riemann surface. Discuss the weak uniform convexity of $Q(R)$.

Recall from $\S 0$ that, for every $\psi \in Q(\Delta)$, the value $\langle\phi, \psi\rangle$ is the Gateaux or weak derivative of the $L^{1}$ norm, in the sense that it is equal to

$$
\lim _{t \rightarrow 0} \frac{|\psi+t \phi|_{1}-|\psi|_{1}}{t} .
$$

A stronger concept of differentiability is the following: 
Definition. The $L^{1}$ norm is Fréchet differentiable at $\psi \in S(\Delta)$ if the limit

$$
\langle\phi, \psi\rangle=\lim _{t \rightarrow 0} \frac{|\psi+t \phi|_{1}-|\psi|_{1}}{t}
$$

exists uniformly for all directions $\phi \in S(\Delta)$.

Problem 4. Is the norm on $Q(\Delta)$ Fréchet differentiable at $\psi=1 / \pi \cdot d z^{2}$ ?

The differentiability of the $L^{1}$ norm on $Q(R)$ has been used to show that the Teichmüller metric of a finite type Riemann surface is $C^{1}[\mathrm{R}]$.

Problem 5. Is the Teichmüller metric on universal Teichmüller space a $C^{1}$ metric?

Let $\mathscr{B}$ denote the Bloch space of holomorphic functions $\phi: \Delta \rightarrow \mathrm{C}$ whose Bloch norm

$$
|\phi|_{\mathscr{B}}=\sup _{z \in \Delta} \rho(z)^{-2}|\phi(z)|
$$

is finite. There is a pairing $\langle\langle\cdot, \cdot\rangle\rangle: L_{a}^{1}(\Delta) \times \mathscr{B} \rightarrow \mathbb{C}$ defined by

$$
\langle\langle\phi, \beta\rangle\rangle=\lim _{t \rightarrow 1} \frac{1}{\pi} \cdot \int_{t \Delta} \phi(z) \overline{\beta(z)}|d z|^{2} .
$$

For each $\beta \in \mathscr{B}$, the map $\beta_{*}: L_{a}^{1}(\Delta) \rightarrow \mathbb{C}$, defined by $\beta_{*}(\phi)=\langle\langle\phi, \beta\rangle\rangle$, is linear, and the transformation $\beta \mapsto \beta_{*}$ is an isomorphism from $\mathscr{B}$ onto the dual space $L_{a}^{1}(\Delta)^{*}[\mathrm{~A}]$.

There is a bounded operator $P$ that projects the space $L^{\infty}(\Delta)$ onto $\mathscr{B}$, defined by

$$
P f(z)=\frac{1}{\pi} \cdot \int_{\Delta} f(w)(1-\bar{w} z)^{-2}|d w|^{2} .
$$

Then $P: L_{\infty}(\Delta) \rightarrow \mathscr{B}$ is analogous to $T: L^{1}(\Delta) \rightarrow L_{a}^{1}(\Delta)$ defined in $\S 1$; in particular, $P(\phi)=\phi$ if $\phi$ is holomorphic. Identifying $L_{a}^{1}(\Delta)$ with $Q(\Delta)$, we obtain a composite map from $S(\Delta)$ to $L^{\infty}(\Delta)$ to $\mathscr{B}$ given by

$$
\psi \rightarrow \psi /|\psi| \rightarrow P(\psi /|\psi|) .
$$

This composition maps $S(\Delta)$ to the Bloch space.

Problem 6. Which Bloch maps (or equivalently, which elements of $L_{a}^{1}(\Delta)^{*}$ ) arise by this construction?

A straightforward calculation with Taylor series shows that

$$
P \frac{\psi}{|\psi|_{*}}(\phi)=\left\langle\left\langle\phi, P \frac{\psi}{|\psi|}\right\rangle\right\rangle=\int_{\Delta} \phi(z) \frac{\overline{\psi(z)}}{|\psi(z)|}|d z|^{2},
$$

which is just our original pairing $\langle\phi, \psi\rangle$ before taking the real part. Thus, the issue in Problem 6 is to analyze the operator $P: L_{\infty}(\Delta) \rightarrow \mathscr{B}$.

\section{ACKNOWLEDGMENT}

The author is grateful to Sheldon Axler, Peter Jones, Curt McMullen, Geoff Mess, and Charles Pugh for enlightening conversations about the material in this article. 


\section{REFERENCES}

[A] S. Axler, Bergman spaces and their operators, Surveys of Some Recent Results in Operator Theory (J. Conway and B. Morrel, eds.), Pitman Res. Notes Math. Ser., vol. 171, Longman Sci. Tech., Harlow, 1988.

[D] J. Diestel, Geometry of Banach spaces-selected topics, Springer Verlag, New York, 1975.

[Ga] F. P. Gardiner, Teichmüller theory and quadratic differentials, Wiley, New York, 1987.

[FR] R. Forelli and W. Rudin, Projections on spaces of holomorphic functions in balls, Indiana Univ. Math. J. 24 (1974), 593-602.

[Mc] C. T. McMullen, Amenability, Poincaré series and quasiconformal maps, Invent. Math. 97 (1989), 95-127.

[R] H. L. Royden, Automorphisms and isometries of Teichmüller space, Ann. of Math. Stud., vol. 66, Princeton Univ. Press, Princeton, NJ, 1971, pp. 368-384.

[S] K. Strebel, Quadratic differentials, Springer-Verlag, New York, 1984.

Department of Mathematics, University of California at Berkeley, Berkeley, CaliFORNIA 94720

E-mail address: goldberg@math.berkely.edu

Brooklyn College and the Graduate Center, City University of New York, BROOKLYN, NEW YORK 11210 